\title{
Four-fermi anomalous dimensions with adjoint fermions
}

\section{Luigi Del Debbio*}

Higgs Centre for Theoretical Physics, The University of Edinburgh, Edinburgh, UK

E-mail: luigi.del.debbioded.ac.uk

\section{Liam Keegan}

PH-TH, CERN, CH-1211 Geneva 23, Switzerland

E-mail: liam.keegan@cern.ch

\section{Carlos Pena}

Instituto de Física Teórica UAM/CSIC

c/Nicolás Cabrera 13-15, Universidad Autónoma de Madrid

Cantoblanco E-28049 Madrid, Spain

Departamento de Física Teórica, Universidad Autónoma de Madrid

Cantoblanco E-28049 Madrid, Spain

E-mail: carlos.pena@uam.es

The four-fermi interaction can play an important role in models of strong dynamical EW symmetry breaking if the anomalous dimensions of the four-fermi operators become large in the IR. We discuss a number of issues that are relevant for the nonperturbative computation of the fourfermi anomalous dimensions for the SU(2) gauge theory with two flavors of Dirac fermions in the adjoint representation, using a Schrödinger functional formalism.

The 32nd International Symposium on Lattice Field Theory,

23-28 June, 2014

Columbia University New York, NY

${ }^{*}$ Speaker. 


\section{Introduction}

The discovery of a Higgs boson begs for a dynamical explanation of the mechanism of electroweak symmetry breaking (EWSB), which seems to be well described by the Higgs sector of the Standard Model. The traditional models of physics beyond the Standard Model (BSM), like minimal supersymmetry, or composite Higgs models, need to be finely tuned in order to agree with the first measurements of the Higgs mass, quantum numbers, and couplings.

New dynamics is needed in order to explain the observed phenomenology. It is conceivable that this new dynamics is dictated by the existence of a strongly-interacting IR fixed point of a gauge theory describing BSM physics.

This possibility can only be explored by developing new, effective tools to study the nonperturbative regime of gauge theories. In a series of papers, we have advocated the usage of lattice simulations to identify possible fixed points, and to characterize them by computing the anomalous dimensions at the fixed point $[1,2,3,4]$. In particular, the value of the anomalous dimension for four-fermi operators is an important ingredient in the description of IR fixed points, and may play an important role for building models of dynamical EWSB [5, 6, 7, 8, 9, 10]

When searching for fixed points, it has become customary to investigate gauge theories with fermions in representations of the color group other than the fundamental one. In particular several studies for the SU(2) theory with two flavors of fermions in the adjoint representation yielded results that are compatible with the existence of an IR fixed point, see e.g. Ref. [11] and references therein for a recent review.

The anomalous dimensions in the neighbourhood of a strongly-interacting IRFP can only be studied in suitably defined nonperturbative renormalization schemes. While the running of the coupling depends on the choice of the scheme, the value of the anomalous dimensions at the fixed point is scheme independent. Here we discuss the computation of the four-fermi anomalous dimension using a Schrödinger functional formalism. Results for the running of the coupling, and the mass anomalous dimension have already been obtained in this scheme [1].

In this note we focus on some of the technical issues that arise when fermions in the adjoint representation are used. Following closely the techniques developed for fermions in the fundamental representation, we highlight the new technical problems that arise and discuss their solution.

\section{Renormalization pattern for adjoint fermions}

The anomalous dimensions of four-fermi operators are extracted from the scale-dependent part of the renormalization constants for these operators. Under renormalization a composite operator mixes with all operators of lower or equal dimension that have the same transformation properties under the symmetries of the theory. Given a regulated theory, a bare composite operator $O$ is renormalized as follows:

- the operators that have the same transformation properties as $O$ under the symmetries unbroken by the regulator are split into one set $\left\{O_{k}\right\}$ of operators that have the same transformation properties as $O$ under all symmetries, including the ones broken by the regulator, and the set of the remaining ones, denoted $\left\{\tilde{O}_{n}\right\}$. 
- operators $O_{k}+c_{k n} \tilde{O}_{n}$ are constructed that have the desired transformation properties under the unbroken symmetries of the renormalized theory. The coefficients $c_{k n}$ depend on the bare coupling and mass. The mass dependence can be constrained using spurionic symmetry.

- in a gauge theory these operators diverge logarithmically only, and the linear combinations $\hat{O}_{j}=Z_{j k}\left[O_{k}+c_{k n} \tilde{O}_{n}\right]$ are finite as the cutoff is removed.

We shall adopt here a mass-independent renormalization scheme, so that the renormalization constants can be computed in the massless limit, and yield anomalous dimensions that are independent of the masses. Note that in order to extract the anomalous dimension, only the divergent part of the renormalization constant is needed.

\section{Mixing with adjoint fermions}

The mixing pattern of four-fermi operators has been studied in detail for fermions in the fundamental representation of the gauge group, see e.g. Ref. [12]. In order to strictly avoid mixing with lower-dimensional operators, we will consider fermions with different flavors that are indicated by the suffix ranging from 1 to 4 . Taking gauge invariance into account, a convenient basis for the description of the operator mixing is given by the set of operators:

$$
\left\{\left(\bar{\psi}_{1} \Gamma_{1}^{(r)} \psi_{2}\right)\left(\bar{\psi}_{3} \Gamma_{2}^{(r)} \psi_{4}\right),\left(\bar{\psi}_{1} \Gamma_{1}^{(r)} T^{A} \psi_{2}\right)\left(\bar{\psi}_{3} \Gamma_{2}^{(r)} T^{A} \psi_{4}\right)\right\}
$$

where the parentheses indicate the trace over spin and color. The set of matrices $\Gamma_{1}^{(r)} \otimes \Gamma_{2}^{(r)}$, for $r=1, \ldots, 10$, spans the set of all Lorentz-invariant spin structures. They can be divided into parityeven and parity-odd operators:

$\begin{array}{cc}\text { parity-even } & \text { parity-odd } \\ \gamma_{\mu} \otimes \gamma_{\mu} & \gamma_{\mu} \otimes \gamma_{\mu} \gamma_{5} \\ \gamma_{\mu} \gamma_{5} \otimes \gamma_{\mu} \gamma_{5} & \gamma_{\mu} \gamma_{5} \otimes \gamma_{\mu} \\ \mathbf{1} \otimes \mathbf{1} & \mathbf{1} \otimes \gamma_{5} \\ \gamma_{5} \otimes \gamma_{5} & \gamma_{5} \otimes \mathbf{1} \\ \sigma_{\mu \nu} \otimes \sigma_{\mu v} & \sigma_{\mu v} \otimes \tilde{\sigma}_{\mu v}\end{array}$

From now on we will use the straightforward notations VV, AA, SS, PP, TT; VA, AV, SP, PS, TT̃ for the above operators.

In this note we focus on the specific issues that arise when working with fermions in the adjoint representation of the gauge group. For fundamental fermions the generators of the gauge group in Eq. 3.1 can be eliminated using the trace identity:

$$
\left(T^{A}\right)_{\alpha \beta}\left(T^{A}\right)_{\gamma \delta}=\frac{1}{2} \delta_{\alpha \delta} \delta_{\beta \gamma}-\frac{1}{2 N} \delta_{\alpha \beta} \delta_{\gamma \delta},
$$

and a Fierz rearrangement of the resulting expressions. These are the usual Fierz identities in the socalled particle-antiparticle channel. As a result only operators of the form $\left(\bar{\psi}_{1} \Gamma_{1}^{(r)} \psi_{2}\right)\left(\bar{\psi}_{3} \Gamma_{2}^{(r)} \psi_{4}\right)$, and $\left(\bar{\psi}_{1} \Gamma_{1}^{(r)} \psi_{4}\right)\left(\bar{\psi}_{3} \Gamma_{2}^{(r)} \psi_{2}\right)$, which are diagonal in color, are required when working in the fundamental representation. 
The trace identity Eq. 3.3 depends on the fermion representation. In particular the corresponding identity for fermions in the adjoint representation of $\mathrm{SU}(2)$ is:

$$
\left(T^{A}\right)_{\alpha \beta}\left(T^{A}\right)_{\gamma \delta}=\delta_{\alpha \delta} \delta_{\beta \gamma}-\delta_{\alpha \gamma} \delta_{\beta \delta}
$$

The structure of the color indices in the right-hand side of this equation suggests that the Fierz identities in the particle-particle channel are also needed in order to rewrite the operators containing the generators $T^{A}$. As a result, the following operators form a basis for describing the mixing of dimension six four-fermi operators:

$$
\begin{aligned}
& O_{\Gamma_{1} \Gamma_{2}}^{(1)}=\left(\bar{\psi}_{1} \Gamma_{1} \psi_{2}\right)\left(\bar{\psi}_{3} \Gamma_{2} \psi_{4}\right), \\
& O_{\Gamma_{1} \Gamma_{2}}^{(2)}=\left(\bar{\psi}_{1} \Gamma_{1} \psi_{4}\right)\left(\bar{\psi}_{3} \Gamma_{2} \psi_{2}\right), \\
& O_{\Gamma_{1} \Gamma_{2}}^{(3)}=\left(\bar{\psi}_{1} \Gamma_{1} C \bar{\psi}_{3}^{T}\right)\left(\psi_{2}^{T} C \Gamma_{2} \psi_{4}\right), \\
& O_{\Gamma_{1} \Gamma_{2}}^{(4)}=\left(\bar{\psi}_{1} \Gamma_{1} C \bar{\psi}_{3}^{T}\right)\left(\psi_{4}^{T} C \Gamma_{2} \psi_{2}\right),
\end{aligned}
$$

where $\Gamma_{1} \otimes \Gamma_{2}$ is any of the spin structures in Eq. 3.2.

The operators $O^{(3)}$ and $O^{(4)}$ are the same up to a sign that depends on the spin structure involved. However, for bookkeeping purposes, it is convenient to keep the operators $O^{(4)}$ in the basis, and only remove them at the end of the discussion.

The transformation properties of these operators under a set of discrete symmetries of the action allow us to constrain the mixing pattern further. The symmetries used here are charge conjugation, parity, and the exchange of flavor indices. They are denoted respectively by $C, P$, and $S_{k l}$. The latter denotes the exchange of flavor $k$ and $l$, while all other flavors are left unchanged.

As discussed above, the basis operators split into a parity-even and a parity-odd sector. The mixing pattern in the parity-odd sector is deduced by classifying the operators according to their transformation properties under $S_{24}, \mathscr{C}_{1234}=C S_{12} S_{34}$, and $\mathscr{C}_{1423}=C S_{14} S_{23}$. We obtain the following sets of operators, characterized by the eigenvalues of $\mathscr{C}_{1234}$ and $\mathscr{C}_{1423}$, and closed under renormalization.

- The sector $\left(\mathscr{C}_{1234}=-1, \mathscr{C}_{1423}=-1\right)$ contains four operators:

$$
\begin{aligned}
& Q_{1}^{ \pm}=Q^{(1)}[V A-A V] \pm Q^{(2)}[V A-A V] \\
& A_{1}^{ \pm}=Q^{(3)}[S P-P S] \pm Q^{(4)}[S P-P S]
\end{aligned}
$$

The superscript \pm indicates operators that are respectively even and odd under $S_{24}$. Under renormalization:

$$
\left(\begin{array}{l}
Q_{1} \\
A_{1}
\end{array}\right)_{R}^{ \pm}=\left(\begin{array}{ll}
Z_{Q_{1} Q_{1}} & Z_{Q_{1} A_{1}} \\
Z_{A_{1} Q_{1}} & Z_{A_{1} A_{1}}
\end{array}\right)^{ \pm}\left(\begin{array}{c}
Q_{1} \\
A_{1}
\end{array}\right)^{ \pm}
$$

where the suffix $R$ denotes the renormalized operators. It is easy to check that $A_{1}^{-}=0$, and therefore the operator $Q_{1}^{-}$renormalizes multiplicatively. 
- The sectors $\left(\mathscr{C}_{1234}=-1, \mathscr{C}_{1423}=+1\right)$, and $\left(\mathscr{C}_{1234}=+1, \mathscr{C}_{1423}=-1\right)$ contain eight operators that can be recombined into eigenstates of $S_{24}$ :

$$
\begin{aligned}
Q_{2}^{ \pm} & =Q^{(1)}[V A+A V] \pm Q^{(2)}[V A+A V] \\
A_{2}^{ \pm} & =Q^{(3)}[V A+A V] \pm Q^{(4)}[V A+A V] \\
Q_{3}^{ \pm} & =Q^{(1)}[S P-P S] \pm Q^{(2)}[S P-P S] \\
A_{3}^{ \pm} & =Q^{(3)}[V A-A V] \pm Q^{(4)}[V A-A V] .
\end{aligned}
$$

The even and odd sectors under $S_{24}$ do not mix, leading to the following mixing matrices:

$$
\left(\begin{array}{l}
Q_{2} \\
A_{2} \\
Q_{3} \\
A_{3}
\end{array}\right)_{R}^{ \pm}=\left(\begin{array}{llll}
Z_{Q_{2} Q_{2}} & Z_{Q_{2} A_{2}} & Z_{Q_{2} Q_{3}} & Z_{Q_{2} A_{3}} \\
Z_{A_{2} Q_{2}} & Z_{A_{2} A_{2}} & Z_{A_{2} Q_{3}} & Z_{A_{2} A_{3}} \\
Z_{Q_{3} Q_{2}} & Z_{Q_{3} A_{2}} & Z_{Q_{3} Q_{3}} & Z_{Q_{3} A_{3}} \\
Z_{A_{3} Q_{2}} & Z_{A_{3} A_{2}} & Z_{A_{3} Q_{3}} & Z_{A_{3} A_{3}}
\end{array}\right)^{ \pm}\left(\begin{array}{c}
Q_{2} \\
A_{2} \\
Q_{3} \\
A_{3}
\end{array}\right)^{ \pm} .
$$

- The sector $\left(\mathscr{C}_{1234}=+1, \mathscr{C}_{1423}=+1\right)$ contains the remaining eight operators. They can be rearranged in eigenstates of $S_{24}$ :

$$
\begin{aligned}
& Q_{4}^{ \pm}=Q^{(1)}[S P+P S] \pm Q^{(2)}[S P+P S] \\
& A_{4}^{ \pm}=Q^{(3)}[S P+P S] \pm Q^{(4)}[S P+P S] \\
& Q_{5}^{ \pm}=Q^{(1)}[T \tilde{T}] \pm Q^{(2)}[T \tilde{T}] \\
& A_{5}^{ \pm}=Q^{(3)}[T \tilde{T}] \pm Q^{(4)}[T \tilde{T}] .
\end{aligned}
$$

The matrix describing the mixing of these operators under renormalization is similar to the one in Eq. 3.13.

\section{Correlators in the Schrödinger functional scheme}

The anomalous dimensions are evaluated by computing the scale dependence of the renormalization constants in the Schrödinger functional (SF) renormalization scheme [13]. We use the implementation of the SF introduced in Refs. [14], adapted for adjoint fermions in Ref. [1]. We use the standard Wilson action for fermions in the adjoint, following Ref. [15].

The strategy to compute the anomalous dimensions is analogous to the one used for fundamental fermions in Refs. [16, 17], which requires the computation of correlators of a four-fermi operator $\mathscr{Q}$ with boundary fields $\mathscr{O}$ and $\mathscr{O}^{\prime}$ :

$$
\int d^{3} x\left\langle\mathscr{O}_{53}^{\prime}\left[\Gamma_{C}\right] \mathscr{Q}(x) \mathscr{O}_{21}\left[\Gamma_{A}\right] \mathscr{O}_{45}\left[\Gamma_{B}\right]\right\rangle
$$

The boundary fields $\mathscr{O}$ and $\mathscr{O}^{\prime}$ are:

$$
\begin{aligned}
\mathscr{O}_{a b}[\Gamma] & =\sum_{\mathbf{y}, \mathbf{z}}\left(\bar{\zeta}_{a}(\mathbf{x}) \Gamma \zeta_{b}(\mathbf{z})\right), \\
\mathscr{O}^{\prime}{ }_{a b}[\Gamma] & =\sum_{\mathbf{y}, \mathbf{z}}\left(\bar{\zeta}_{a}^{\prime}(\mathbf{x}) \Gamma \zeta_{b}^{\prime}(\mathbf{z})\right),
\end{aligned}
$$


where the primed and unprimed quantities refer to fields at the $t=L$ and $t=0$ boundary respectively. These correlators are all constructed from contractions of bulk-to-boundary correlators, denoted $H(x)$ and $H^{\prime}(x)$, and boundary-to-boundary correlators, denoted by $\mathscr{H}$. Explicit expressions for these correlators can be found in Ref. [18]. For $\mathscr{Q}=\left(\bar{\psi}_{1} \Gamma_{1} \psi_{2}\right)\left(\bar{\psi}_{3} \Gamma_{2} \psi_{4}\right)$, and $\mathscr{Q}=\left(\bar{\psi}_{1} \Gamma_{1} \psi_{4}\right)\left(\bar{\psi}_{3} \Gamma_{2} \psi_{2}\right)$ the contractions required are identical to the ones that appear with fundamental fermions. For instance, for the latter four-fermi operator, the correlator in Eq. 4.1 is given by:

$$
W_{2}=-\int d^{3} x\left\langle\operatorname{tr}\left[\gamma_{5} H_{1}(x)^{\dagger} \gamma_{5} \Gamma_{1} H_{4}(x) \Gamma_{B} \mathscr{H}_{5} \Gamma_{C} \gamma_{5} H_{3}^{\prime}(x)^{\dagger} \gamma_{5} \Gamma_{2} H_{2}(x) \Gamma_{A}\right]\right\rangle_{G} .
$$

However, because we are now working with fermions in the adjoint representation, the operators $\mathscr{Q}$ span the wider basis discussed in the previous Section, which suggests that new correlators are needed that were not computed for the case of fundamental fermions. These are the correlators where:

$$
\mathscr{Q}=\left(\bar{\psi}_{1} \Gamma_{1} C \bar{\psi}_{3}^{T}\right)\left(\psi_{4}^{T} \Gamma_{2} \psi_{2}\right) .
$$

It can be shown that these operators give rise to contractions that are identical to the ones needed for fundamental fermions, up to permutations of the matrices that determine the spin structure of the operator $\mathscr{Q}$. For example, the operator $\mathscr{Q}$ in Eq. 4.5 leads to:

$$
W_{3} \propto \int d^{3} x\left\langle\operatorname{tr}\left[\gamma_{5} H_{1}(x)^{\dagger} \gamma_{5} \Gamma_{1} H_{\tilde{3}}^{\prime}(x) \Gamma_{C}\left(\gamma_{5} \mathscr{H}_{\tilde{5}} \gamma_{5}\right) \Gamma_{B} \gamma_{5} H_{\tilde{4}}(x)^{\dagger} \gamma_{5} \Gamma_{2} H_{2}(x) \Gamma_{A}\right]\right\rangle_{G},
$$

where we introduced the propagator for the charge conjugate flavor:

$$
\begin{aligned}
C H_{f}(x)^{T} C & =\gamma_{5} H_{\tilde{f}}^{\dagger} \gamma_{5}, \\
C\left(\gamma_{5} H_{f}(x)^{\dagger} \gamma_{5}\right)^{T} C & =H_{\tilde{f}}(x) .
\end{aligned}
$$

The expression for $W_{3}$ is readily related to the contractions that appear in $W_{2}$ above, after swapping $\Gamma_{1}$ and $\Gamma_{2}$. Having computed all the possible contractions for the $W_{2}$ trace, $W_{3}$ can be reconstructed without further computations. Therefore all the ingredients are in place to complete the numerical investigation of the anomalous dimension.

\section{Conclusion}

In this note we have presented some of the peculiar questions that arise when computing anomalous dimensions using the SF and adjoint fermions. We discussed in detail the answer to these questions, showing that the case of adjoint fermions can be dealt with in a way that resembles the solution for the case of fundamental fermions. Extra care is needed when constructing the basis of operators, and when inserting these operators into SF correlators.

A detailed account of our studies is in preparation.

\section{References}

[1] F. Bursa, L. Del Debbio, L. Keegan, C. Pica and T. Pickup, Phys. Rev. D 81, 014505 (2010) [arXiv:0910.4535 [hep-ph]].

[2] L. Del Debbio and R. Zwicky, Phys. Rev. D 82, 014502 (2010) [arXiv:1005.2371 [hep-ph]]. 
[3] L. Del Debbio, B. Lucini, A. Patella, C. Pica and A. Rago, Phys. Rev. D 82, 014510 (2010) [arXiv:1004.3206 [hep-lat]].

[4] S. Catterall, L. Del Debbio, J. Giedt and L. Keegan, Phys. Rev. D 85, 094501 (2012) [arXiv:1108.3794 [hep-ph]].

[5] W. A. Bardeen, C. N. Leung and S. T. Love, Phys. Rev. Lett. 56, 1230 (1986).

[6] V. A. Miransky and K. Yamawaki, Mod. Phys. Lett. A 4, 129 (1989).

[7] K. -i. Kondo, S. Shuto and K. Yamawaki, Mod. Phys. Lett. A 6, 3385 (1991).

[8] D. K. Hong, S. D. H. Hsu and F. Sannino, Phys. Lett. B 597, 89 (2004) [hep-ph/0406200].

[9] M. A. Luty and T. Okui, JHEP 0609, 070 (2006) [hep-ph/0409274].

[10] R. Rattazzi, V. S. Rychkov, E. Tonni and A. Vichi, JHEP 0812, 031 (2008) [arXiv:0807.0004 [hep-th]].

[11] J. Kuti, PoS LATTICE 2013, 004 (2014).

[12] A. Donini, V. Gimenez, G. Martinelli, M. Talevi and A. Vladikas, Eur. Phys. J. C 10, 121 (1999) [hep-lat/9902030].

[13] K. Symanzik, Nucl. Phys. B 190, 1 (1981).

[14] S. Sint, Nucl. Phys. B 421, 135 (1994) [hep-lat/9312079].

[15] L. Del Debbio, A. Patella and C. Pica, Phys. Rev. D 81, 094503 (2010) [arXiv:0805.2058 [hep-lat]].

[16] M. Guagnelli et al. [ALPHA Collaboration], JHEP 0603, 088 (2006) [hep-lat/0505002].

[17] P. Dimopoulos et al. [ALPHA Collaboration], JHEP 0805, 065 (2008) [arXiv:0712.2429 [hep-lat]].

[18] M. Luscher and P. Weisz, Nucl. Phys. B 479, 429 (1996) [hep-lat/9606016]. 